\title{
Bicuspid aortic valve and aortic coarctation in congenital heart disease-important aspects for treatment with focus on aortic vasculopathy
}

\author{
Christoph Sinning ${ }^{1,2 \#}$, Elvin Zengin ${ }^{1 \#}$, Rainer Kozlik-Feldmann ${ }^{3}$, Stefan Blankenberg ${ }^{1,2}$, Carsten Rickers ${ }^{3}$, \\ Yskert von Kodolitsch ${ }^{1}$, Evaldas Girdauskas ${ }^{4}$ \\ ${ }^{1}$ Department of General and Interventional Cardiology, University Heart Center Hamburg, Hamburg, Germany; ${ }^{2}$ German Center of Cardiovascular \\ Research (DZHK), Partner Site Hamburg/Kiel/Lübeck, Germany; ${ }^{3}$ Department of Pediatric Cardiology, ${ }^{4}$ Department of Cardiac and Cardiovascular \\ Surgery, University Heart Center Hamburg, Hamburg, Germany \\ Contributions: (I) Conception and design: C Sinning, E Zengin, Y von Kodolitsch, E Girdauskas; (II) Administrative support: None; (III) Provision \\ of study materials or patients: None; (IV) Collection and assembly of data: C Sinning, E Zengin; (V) Data analysis and interpretation: C Sinning, E \\ Zengin, Y von Kodolitsch, E Girdauskas; (VI) Manuscript writing: All authors; (VII) Final approval of manuscript: All authors. \\ \#These authors contributed equally to this work. \\ Correspondence to: Christoph Sinning, MD. Department of General and Interventional Cardiology, Universtiy Heart Center Hamburg, Martinistr. 52, \\ 20246 Hamburg, Germany. Email: c.sinning@uke.de.
}

\begin{abstract}
Prevalence of congenital heart disease (CHD) is constantly increasing during the last decades in line with the treatment options for patients ranging from the surgical as well to the interventional spectrum. This mini-review addresses two of the most common defects with bicuspid aortic valve (BAV) and coarctation of the aorta (CoA). Both diseases are connected to aortic vasculopathy which is one of the most common reasons for morbidity and mortality in young patients with CHD. The review will focus as well on other aspects like medication and treatment of pregnant patients with BAV and CoA. New treatment aspects will be as well reviewed as currently there are additional treatment options to treat aortic regurgitation or aortic aneurysm especially in patients with valvular involvement and a congenital BAV thus avoiding replacement of the aortic valve and potentially improving the future therapy course of the patients.
\end{abstract}

Keywords: Bicuspid aortic valve (BAV); aortic coarctation; aortic vasculopathy; aortic valve reconstruction

Submitted Jul 23, 2018. Accepted for publication Sep 25, 2018.

doi: $10.21037 / \mathrm{cdt} .2018 .09 .20$

View this article at: http://dx.doi.org/10.21037/cdt.2018.09.20

\section{Introduction}

Bicuspid aortic valve (BAV) is the most common congenital heart defect with a prevalence of $1-2 \%$ and most commonly $\mathrm{BAV}$ is found in males with a rate of 1:2 varying to 1:4 (1-5). $\mathrm{BAV}$ is most commonly the result of fusion of the left and right coronary cusp (LCC and RCC) in over 70\% of patients and not so common of fusion of the RCC with the non-coronary cusp (NCC) 10-20\% and least frequent due to fusion of the LCC with NCC in $5-10 \%(1,3)$.

Coarctation of the aorta $(\mathrm{CoA})$ occurs often as a discrete stenosis or a longer, hypoplastic segment of the ascending aorta. Typically, CoA occurs where the ductus arteriosus is located and only rarely ectopically in the ascending, descending or abdominal aorta (1-3). Most often reported is a prevalence in relation to all congenital heart disease (CHD) of $5-8 \%$ and a prevalence of 3 in 10,000 live births for the isolated form of $\mathrm{CoA}(1,2)$.

Both BAV and CoA as CHD are commonly associated in $85 \%$ of cases and can be present together with subvalvular, valvular or supravalvular aortic stenosis and malformation of the mitral valve with mitral valve stenosis. The combination of aortic stenosis at all three levels with a parachute mitral valve is called Shone complex (1). However, CoA can as well often be found in complex and genetic lesions with Turner 
or Williams-Beuren Syndrome.

An important associated lesion in both above-mentioned diseases is the aortopathy, in both types of CHD. Aortopathy could be a potential factor responsible for the increased morbidity and mortality in both diseases. A specific diagnostic work-up has been reported to consider the need for simultaneous aortic surgery to decrease the risk of potential lethal aortic dissection when aortic valve surgery is required $(1-3,6)$.

This focused mini-review describes the current diagnostic and treatment algorithm for both diseases with a special focus regarding aortopathy. Further, new aspects regarding aortic valve surgery in both diseases will be addressed as these should be considered when making the surgical decision in mostly young patients to achieve the best long-term result.

\section{Methods}

\section{Literature search}

Until May 2018, a literature search was performed in PubMed. The following combinations of keywords were used: bicuspid aortic valve, coarctation of the aorta, aortic vasculopathy, aortic stenosis or regurgitation of bicuspid aortic valve, aortic reconstruction and aortic valve reconstruction. These search terms had to be identified anywhere in the text in the articles. Both qualitative and quantitative studies were considered to elucidate the use of the different aspects regarding BAV, CoA and combination of both diseases. The search was restricted to original research, humans, and papers published in English at any date. All abstracts were reviewed to assess whether the article met the inclusion criteria. The key inclusion criterion was the presence of BAV, CoA or of both diseases according to the diagnostic guidelines at the time of the study. In addition, one of the following criteria had to be fulfilled: one of the diseases was related to the outcome measure; or an outcome distribution was reported for BAV, CoA or the general population in the results section. After this selection process, manual search of the reference lists of all eligible articles was performed. Two authors (i.e., C Sinning and E Zengin) assessed independently the methodological quality of the qualitative and quantitative studies prior to their inclusion in the review.

\section{BAV and management of associated lesions}

BAV is the most common CHD, however only about $7 \%$ of patients with BAV have a concomitant CoA. On the other hand, BAV can be found in $70-75 \%$ of patients with CoA (1-3,7-9). In this context, the LCC-RCC fusion type of BAV was found most often in CoA patients and only few data are available describing the concomitant presence of both diseases. Several investigators reported that simultaneous presence of BAV and CoA is more often associated with aortic dilatation as compared to the isolated BAV or CoA cases (1-3,9-12) (Figure $1 A, B, C)$.

\section{Natural development in patients with BAV regarding aortic vasculature}

Natural change of aortic dimension may vary considerably in BAV patients but a diameter increase of 1-2 mm per year has been most commonly reported (3). However, a rapid progression of the aortopathy (Figure 2) up to $5 \mathrm{~mm}$ per year may occur and could be associated with an increased risk of aortic dissection (3,13-16). The change in aortic diameters is concurrently influenced by the severity of valvular lesion (aortic stenosis or regurgitation) which is present in most BAV patients. Nonetheless, quite a few patients may experience a fast increase in aortic diameters despite echocardiographic normal BAV which is mostly the case in cohorts of younger patients $(7,8,13)$. In cases of BAV, surgery of the ascending aorta is indicated in case of: aortic root or ascending aortic diameter $>45 \mathrm{~mm}$ when surgical aortic valve replacement is scheduled $(1,3)$. However, correction of the valvular disease (stenosis and/ or regurgitation) might ameliorate the progression of the aortopathy (3,17-19).

Current guidelines define the presence of aortic aneurysm at aortic diameter $\geq 40 \mathrm{~mm}$ or indexed aortic diameter $\geq 27.5 \mathrm{~mm} / \mathrm{m}^{2}$ in subjects with a short stature and thus small body surface area. The distribution of the different BAV fusion types are of relevance regarding the form of resulting aortic dilation. In the LCC-RCC fusion type of BAV the ascending aorta is dilated most commonly including a frequent involvement of the aortic root (3). In RCC-NCC fusion type predominant dilation of the distal ascending aorta is common, while involvement of the aortic root does not appear to be frequent.

Bicuspid aortopathy occurs most commonly at the level of the tubular ascending aorta with a progression rate of about $0.5 \mathrm{~mm}$ per year which is much slower as compared to the patients with a Marfan syndrome (20). However, no definite prognostic factors of rapid aortic progression have been identified yet and a significant proportion of BAV 

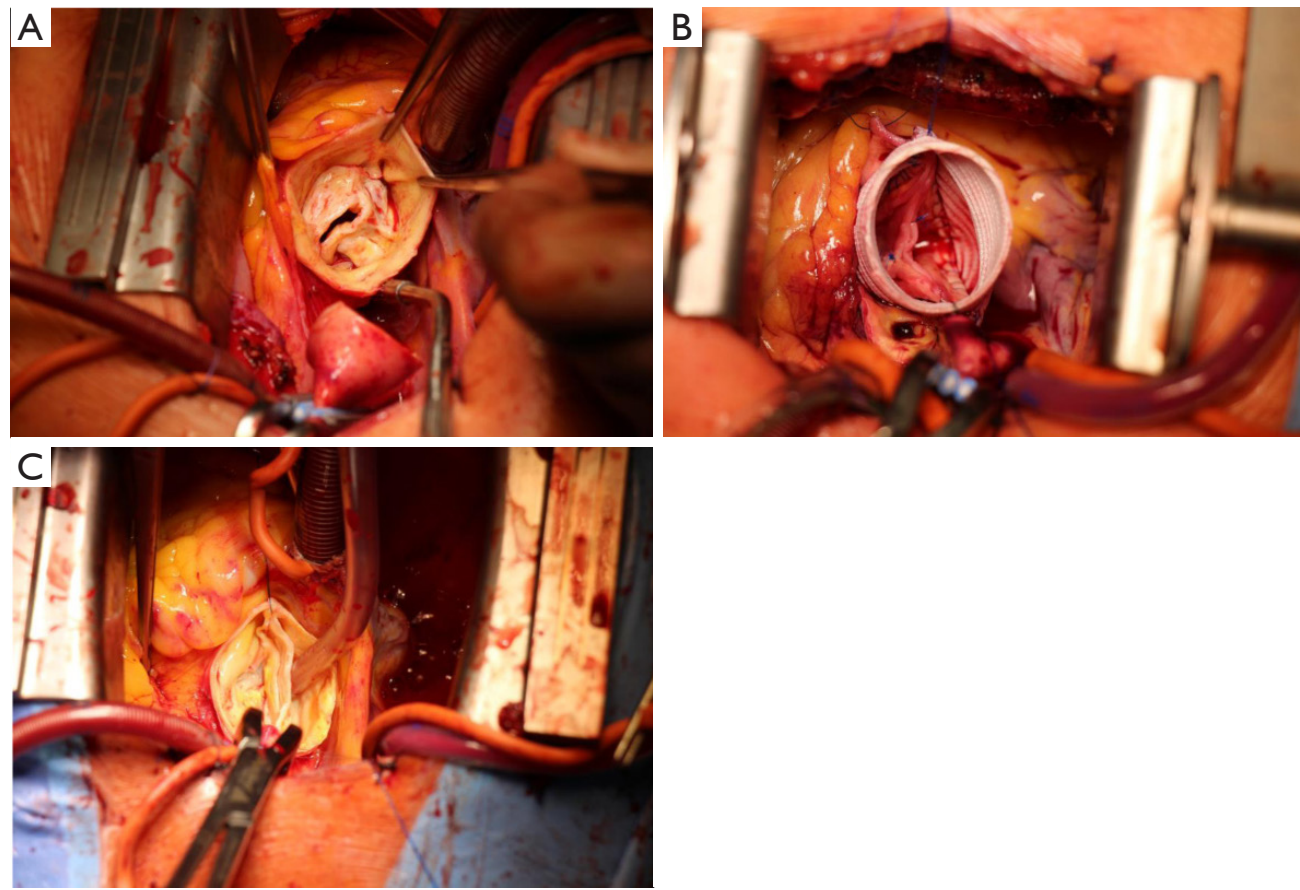

Figure 1 Different types of aortic valve morphology with (A) unicuspid valve, (B) bicuspid aortic valve after reconstruction and (C) bicuspid valve.

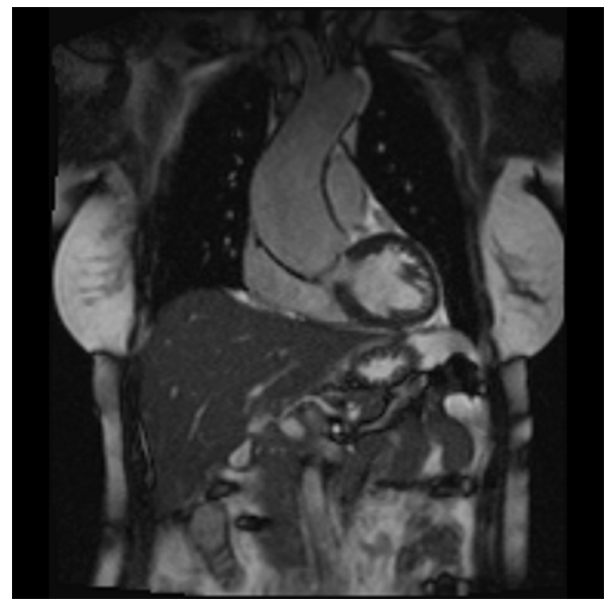

Figure 2 An example of aortopathy with aneurysm of the ascending aorta.

patients have no increase in aortic diameter for decades. Thus, BAV patients are a very heterogenous population and a reliable prediction of concomitant aortopathy is currently not possible $(3,21)$. Even patients with echocardiographic normal valves and diagnosis of BAV will require cardiovascular surgery in about $27 \%$ of the cases over a period of 20 years of follow-up $(3,21)$.

\section{Pathophysiology in patients with BAV}

NOTCH1 gene mutation were most frequently reported in BAV patients. The mode of inheritance resembles autosomal dominant pattern with a reduced penetrance of the disease $(22,23)$. Some previous studies suggested that different types of BAV are associated with different types of aortopathy although the exact pathophysiological pathways are currently unknown $(3,22,23)$. It has been hypothesized that potential common genetic pathways exist for BAV and aortic dilatation or, alternatively, aortic dilation might be a result of abnormal transvalvular flow-patterns in BAV patients. Most authors concluded that both pathogenetic pathways might be contributing to the aortic changes which are reported in this disease spectrum $(3,24,25)$.

\section{Diagnosis of BAV and presence of aortic dilation}

Although BAV is often associated with aortic stenosis or aortic regurgitation or the combination of both it can be suspected during clinical examination by means of auscultation while detecting a heart murmur or symptoms 
of compression of thoracic structures by marked aortic aneurysm (tracheal compression or compression of the laryngeal nerve with corresponding inspiratory stridor or hoarseness). It should be noted, however that in contrast to reports under ideal study conditions, transthoracic echocardiography may yield a sensitivity for BAV of less than $50 \%$ under clinical routine conditions (26). If acoustic window allows for a high-quality examination, the BAV fusion type and functional valvular lesion (relevant aortic stenosis or regurgitation) can be reliably identified. However, it is important to point out the fact that continuity equation cannot reliably used in BAV patients due to altered valve geometry $(27,28)$. Furthermore, in $\mathrm{BAV}$ patients with aortic regurgitation, the regurgitant jet is often eccentric due to the congenital pathology of the valve with the resulting difficulty to use some of the conventional echocardiographic criteria to grade the regurgitation (3). Upon detection of BAV, simultaneous echocardiographic evaluation of the aortic root is feasible and reproducible, however, should be combined with magnetic resonance imaging (MRI) at the time of diagnosis in order to obtain baseline values of the aorta for subsequent follow-up examinations. If the maximal diameter of the ascending aorta reaches the limit of $45 \mathrm{~mm}$, annual follow-up and imaging examinations by means of echocardiography or MRI is recommended. An additional second imaging modality (MRI or CT scan) should be as well used in those patients with an increase of aortic diameters $\geq 3 \mathrm{~mm} /$ year as measured by echocardiography so that high-risk patients can be identified (3).

\section{Treatment of valvular defects in BAV and aortic dilation}

Although there are no studies of isolated medical treatment in patients with $\mathrm{BAV}$ and aortic dilation, often beta blocker therapy is started in patients with aortic dilation to lower the blood pressure (3). Medical treatment of valvular defects like stenosis or regurgitation is only symptomatic and ameliorates symptoms (29-31). For patients with a valvular defect and/or aortic dilation the only curative therapy is surgery.

Several previous studies have shown that patients with progressive dilation of the left ventricle and with only slightly impaired ejection fraction in the setting of aortic regurgitation have a considerable risk of mortality during follow-up $(32,33)$. Thus, the highly controversial issue is whether the patients with BAV and severe aortic regurgitation should be treated even without depressed ejection fraction or with beginning dilation of the left ventricular diameter. In previous years mechanical prostheses were the treatment of choice in severe aortic regurgitation and stenosis in BAV patients or as an oftendiscussed alternative biological prosthesis of the aortic valve. However, both treatment options have a considerable tradeoff for the patient with anticoagulation, thrombus formation or endocarditis especially in patients with mechanical prosthesis $(34,35)$. Although anticoagulation is not needed in biological prosthesis regularly, the need for redo-surgery or valve-in-valve implantation due to ongoing degeneration of the tissue valve prosthesis should not be neglected (36-38). As a consequence, in the recent years surgery of the aortic valve more often uses reconstructive techniques with good results diminishing the need for redo-operations with a low risk of endocarditis as well $(39,40)$. Thus, reconstruction of the ascending aorta and of the aortic valve should be considered when a patient has definite high-grade aortic regurgitation especially in conjunction with the need for surgery due to aortic dilation (39-41). Thus, currently there is a change of the paradigm that valvular defects should be treated by mechanical valves in young BAV patients because this cohort of patients is most likely to develop complications and most probably needs another surgical intervention over time. Regarding the recommendation to operate isolated aortic dilation (i.e., without concomitant valvular lesion), $55 \mathrm{~mm}$ is considered the border for surgery in patients with BAV and without additional risk factors as arterial hypertension, family history of aortic dissection or progression of dilation above $3 \mathrm{~mm}$ per year. The cut-off in patients with above mentioned risk factors is $50 \mathrm{~mm}$, while $45 \mathrm{~mm}$ cut-off value is common for patients if concomitant surgery of the aortic valve is scheduled (3).

\section{Pregnancy in patients with BAV and aortic dilation}

Although BAV is more often diagnosed in men, it is not uncommon in women as well. If diagnosed in patients during pregnancy or with the wish for children, different aspects must be considered before giving the patient a reliable recommendation regarding the risk of pregnancy. Most commonly the risk stratifications scheme of the World Health Organization (WHO) is used to identify patients with an increased risk in class 1 or 2 to patients with a considerable risk (3) to high risk were pregnancy is not recommended in class 4 (42). Regarding aortic dilation the 


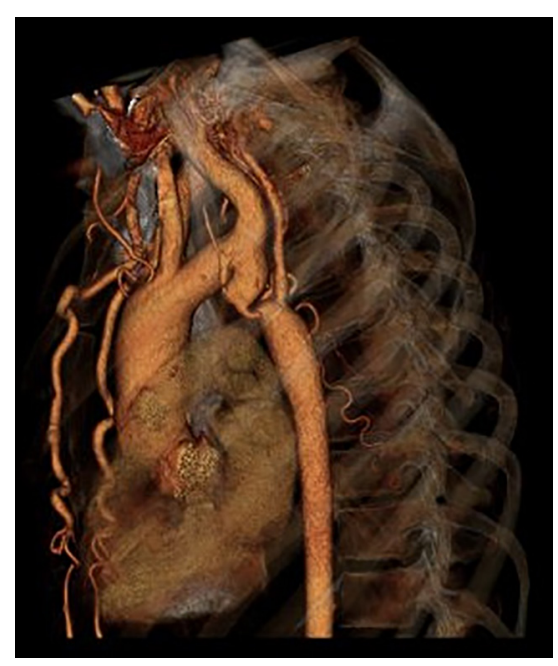

Figure 3 Computer tomography reconstruction of coarctation of the aorta.

cut-off value of $50 \mathrm{~mm}$ of the ascending aorta is used most commonly to define the patients at high-risk which should have surgery before pregnancy the same is true for severe aortic regurgitation as well as for severe aortic stenosis (42). However, BAV with aortopathy is most often asymptomatic and thus not known before pregnancy. Therefore, the most common clinical scenario is new diagnosis of BAV and/or aortic dilation during pregnancy. If severe valvular defects or aortic dilation above the cut-off of $50 \mathrm{~mm}$ is discovered by chance, the patient should have very close monitoring throughout the pregnancy with at least one visit during each trimester. Interdisciplinary discussion with the obstetricians should clarify whether the delivery of the child has to be done via caesarean section. From cardiological point of view, the indication for caesarean section is present in case of severe aortic stenosis or dilation of ascending aorta above $50 \mathrm{~mm}$. However, every pregnancy should be evaluated individually, and some patients will decide for pregnancy despite high risk of cardiovascular events and have to be monitored and treated by an interdisciplinary team of various medical disciplines to ensure optimum therapy (42).

\section{CoA and associated lesions of the aortic vasculature}

$\mathrm{CoA}$ is considered as a generalized arteriopathy rather than only a short stenosis in the region of the insertion of the ductus arteriosus in the aorta (Figure 3). The presence of relevant CoA results in a significant increase of the afterload of the left ventricle in combination with an increased wall stress and thus compensatory left ventricular hypertrophy which may lead to subsequent left ventricular dysfunction. In patients with $\mathrm{CoA}$ alterations of the aortic wall in the ascending and descending aorta were described resulting in an increased stiffness of the aorta and as well of the carotid arteries $(1-3,43)$.

The most prominent symptoms which are often encountered in patients with relevant $\mathrm{CoA}$ are complaints of dizziness, headache, nose bleeds as a result of the severe arterial hypertension which often cannot be reliably controlled with medication. Additionally, various combination of symptoms of malperfusion of the lower body may occur (e.g., abdominal angina, claudication, leg cramps and cold feet) (1-3). As a consequence of this hemodynamic load, the patients might be subject of left heart failure, intracranial hemorrhage from aneurysms in the cerebral circulation, aortic dissection and manifestation of severe coronary artery disease at very young age.

\section{Diagnostic procedures in patients with CoA or suspected CoA}

The predominant sign during clinical examination is arterial hypertension in the upper body, especially upon measurement at the right arm (due to the fact that the right subclavian artery has its take-off proximally of the ductus arteriosus) in comparison to the measurement at the left arm which is supplied by blood from the left subclavian artery and thus from an artery behind the insertion of the ductus arteriosus. In case of clinically relevant CoA an arterial pressure gradient of at least $20 \mathrm{mmHg}$ between the upper and the lower extremities is recorded. Additional information could be obtained by auscultation with a suprasternal thrill and vascular murmur can be heard (44-46).

Echocardiography is the imaging modality of first choice which helps to visualize the site of the coarctation, its length and potential association with other cardiac pathologies (e.g., decreased left ventricular function, left ventricular hypertrophy or presence of BAV). Doppler gradients might be diagnostic but are not reliable as in case of significant collaterals pressure gradient might be absent before and after intervention in the region of the CoA. Thus, the most reliable sign of a hemodynamically significant $\mathrm{CoA}$ is a diastolic run-off (1). In this context it has to be pointed out that after interventional or surgical repair of the CoA the measured gradient can be increased because of the reduced vessel compliance and an increased arterial stiffness in 
patients with CoA. To evaluate the whole aorta in patients with CoA, the use of MRI or CT is recommended, so that aneurysms in different sites of the aorta can be evaluated as well as a potential restenosis after previous interventional/ surgical treatment (1).

Cardiac catheterization is still used in many centers and indicates a relevant CoA if peak-to-peak gradient $\geq 20 \mathrm{mmHg}$ is measured in combination with an absence of relevant collateral arteries. Simultaneous coronary angiography might be reasonable to evaluate the coronary arteries and to exclude relevant coronary artery disease which may impact the treatment strategy of the patient $(47,48)$. However, recent studies could show that $\mathrm{CoA}$ alone is not a risk factor for relevant coronary artery disease (49).

\section{Surgical vs. interventional treatment of patients with CoA}

In the background of improving interventional techniques, primary stenting of the CoA is currently the treatment of choice if the corresponding anatomy is favorable $(1,47,48)$. This is also true for recurrent CoA or residual CoA detected during the follow-up examination of patients with previously treated CoA $(1,47,48)$. Although good surgical results were reported (44-46) decreased procedural risk and faster mobilization after interventional treatment led to a decreased morbidity and mortality in CoA patients $(1,47,48)$. An exception to this is CoA in patients with Turner syndrome. The affected aorta is especially vulnerable in these patients and interventional treatment might lead to life-threatening complications and therefore, the surgical repair is still preferred in significant $\mathrm{CoA}$ in the presence of Turner syndrome (50).

However, it has to be highlighted that CoA is not a localized disease but a generalized arteriopathy of the whole aorta and thus additional vascular lesions might be present which should be looked for or might be the reason for primary correction of the CoA as well. The most common associated lesions are (I) significant aortic valve disease with stenosis or regurgitation especially if BAV is present; (II) aneurysm of the aorta with a diameter of $50 \mathrm{~mm}$ or larger (or $\geq 27.5 \mathrm{~mm} / \mathrm{m}^{2}$ body surface area); (III) recurrent coarctation at the site of the previous intervention; (IV) Aneurysms in the cerebral circulation (1).

Despite close follow-up strategy, arterial hypertension may remain a major clinical problem which may exist parallel to a relevant CoA (51). Thus, it is advisable to treat the patients accordingly even without the obvious presence of hypertensive blood pressure levels since the lack of hypertension might be related to the severity of the CoA $(1,51)$. Furthermore, follow-up examinations should be scheduled at least every two years in combination with an appropriate imaging (MRI to detect recurrent coarctation or additional pathologies at an early stage).

\section{Pregnancy in the presence of CoA}

Depending on the presence of relevant CoA or residual CoA pregnancy can be tolerated well but there might be problems during the pregnancy if an undetected relevant CoA results in a hypoperfusion of the fetus who is supplied via the placental artery. Thus, it is recommended to survey the patients with known CoA and those after CoA repair or asymptomatic CoA whether specific therapy is needed. Antihypertensive treatment should be modified to the approved antihypertensive treatment regime during the pregnancy, as angiotensin-converting-enzyme inhibitors or angiotensinogen-receptor blockers are contraindicated during the pregnancy. If these factors are well-controlled, the patient has regular visits with her obstetrician and the physician being board-certified for the treatment of adults with CHD, the pregnancy can be safely monitored and mode of delivery can be most commonly spontaneous in all cases $(1,3,52)$.

\section{Summary}

The BAV and CoA are common diseases in the spectrum of CHD and are closely related to aortopathy which must be considered in all decision-making processes. Detection of either BAV and/or CoA should trigger additional screening of the patient as concomitant diseases might be always present. In particular, dilation of the ascending aorta carries an increased risk of dissection in both diseases but is highest upon the combination of both diseases. In BAV which represents the most common congenital heart defect new therapy strategies were introduced which are currently not included into the guidelines. The reconstruction of the native BAV might be an adequate therapy alternative in patients to avoid biological or mechanical valve prostheses. Furthermore, the timing of surgery should be considered at an earlier time point by the interactive institutional heart team to avoid late stages of valvular cardiomyopathy which is often irreversible. An important aspect in all young patients with CHD is the risk of pregnancy and the risk can 
be kept very low for both diseases most commonly if regular follow-ups are scheduled and aortic lesions are treated upon reaching certain cut-off aortic diameters before the pregnancy.

In summary, the management of BAV and CoA underwent major advances during the last decade and resulted in improved long-tern outcome and quality of life of the patients. This mini-review discussed new treatment and disease aspects which should be evaluated in further studies.

\section{Acknowledgements}

We want to remind of the work Dr. Dietmar Koschyk did contribute to the section of congenital heart disease at the University Heart Center Hamburg. Dr. Koschyk died in a car accident in 2017.

\section{Footnote}

Conflicts of Interest: The authors have no conflicts of interest to declare.

\section{References}

1. Baumgartner H, Bonhoeffer P, De Groot NM, et al. ESC Guidelines for the management of grown-up congenital heart disease (new version 2010). Eur Heart J 2010;31:2915-57.

2. Bedard E, Shore DF, Gatzoulis MA. Adult congenital heart disease: a 2008 overview. Br Med Bull 2008;85:151-80.

3. Erbel R, Aboyans V, Boileau C, et al. 2014 ESC Guidelines on the diagnosis and treatment of aortic diseases:

Document covering acute and chronic aortic diseases of the thoracic and abdominal aorta of the adult. The Task Force for the Diagnosis and Treatment of Aortic Diseases of the European Society of Cardiology (ESC). Eur Heart J 2014;35:2873-926.

4. von Kodolitsch Y, Kutsche K. Genetic diagnostics of inherited aortic diseases : Medical strategy analysis. Herz 2017;42:459-67.

5. von Kodolitsch Y, Robinson PN. Marfan syndrome: an update of genetics, medical and surgical management. Heart 2007;93:755-60.

6. Michelena HI, Khanna AD, Mahoney D, et al. Incidence of aortic complications in patients with bicuspid aortic valves. JAMA 2011;306:1104-12.

7. Cripe L, Andelfinger G, Martin LJ, et al. Bicuspid aortic valve is heritable. J Am Coll Cardiol 2004;44:138-43.

8. Huntington K, Hunter AG, Chan KL. A prospective study to assess the frequency of familial clustering of congenital bicuspid aortic valve. J Am Coll Cardiol 1997;30:1809-12.

9. Sievers HH, Schmidtke C. A classification system for the bicuspid aortic valve from 304 surgical specimens. J Thorac Cardiovasc Surg 2007;133:1226-33.

10. Ciotti GR, Vlahos AP, Silverman NH. Morphology and function of the bicuspid aortic valve with and without coarctation of the aorta in the young. Am J Cardiol 2006;98:1096-102.

11. Fernandes S, Khairy P, Graham DA, et al. Bicuspid aortic valve and associated aortic dilation in the young. Heart 2012;98:1014-9.

12. Oliver JM, Alonso-Gonzalez R, Gonzalez AE, et al. Risk of aortic root or ascending aorta complications in patients with bicuspid aortic valve with and without coarctation of the aorta. Am J Cardiol 2009;104:1001-6.

13. Chandra S, Lang RM, Nicolarsen J, et al. Bicuspid aortic valve: inter-racial difference in frequency and aortic dimensions. JACC Cardiovasc Imaging 2012;5:981-9.

14. Della Corte A, Romano G, Tizzano F, et al. Echocardiographic anatomy of ascending aorta dilatation: correlations with aortic valve morphology and function. Int J Cardiol 2006;113:320-6.

15. Kinoshita N, Mimura J, Obayashi C, et al. Aortic root dilatation among young competitive athletes: echocardiographic screening of 1929 athletes between 15 and 34 years of age. Am Heart J 2000;139:723-8.

16. Reed CM, Richey PA, Pulliam DA, et al. Aortic dimensions in tall men and women. Am J Cardiol 1993;71:608-10.

17. Girdauskas E, Disha K, Borger MA, et al. Long-term prognosis of ascending aortic aneurysm after aortic valve replacement for bicuspid versus tricuspid aortic valve stenosis. J Thorac Cardiovasc Surg 2014;147:276-82.

18. Girdauskas E, Disha K, Raisin HH, et al. Risk of late aortic events after an isolated aortic valve replacement for bicuspid aortic valve stenosis with concomitant ascending aortic dilation. Eur J Cardiothorac Surg 2012;42:832-7; discussion 837-8.

19. Girdauskas E, Rouman M, Borger MA, et al. Comparison of aortic media changes in patients with bicuspid aortic valve stenosis versus bicuspid valve insufficiency and proximal aortic aneurysm. Interact Cardiovasc Thorac Surg 2013;17:931-6.

20. Detaint D, Michelena HI, Nkomo VT, et al. Aortic dilatation patterns and rates in adults with bicuspid aortic valves: a comparative study with Marfan syndrome and 
degenerative aortopathy. Heart 2014;100:126-34.

21. Girdauskas E, Rouman M, Disha K, et al. Functional Aortic Root Parameters and Expression of Aortopathy in Bicuspid Versus Tricuspid Aortic Valve Stenosis. J Am Coll Cardiol 2016;67:1786-96.

22. Garg V, Muth AN, Ransom JF, et al. Mutations in NOTCH1 cause aortic valve disease. Nature 2005;437:270-4.

23. Fernandez B, Duran AC, Fernandez-Gallego T, et al. Bicuspid aortic valves with different spatial orientations of the leaflets are distinct etiological entities. J Am Coll Cardiol 2009;54:2312-8.

24. Ouzounian M, LeMaire SA. How can genetic diagnosis inform the decision of when to operate? J Vis Surg 2018;4:68.

25. Rodriguez-Palomares JF, Dux-Santoy L, Guala A, et al. Aortic flow patterns and wall shear stress maps by 4D-flow cardiovascular magnetic resonance in the assessment of aortic dilatation in bicuspid aortic valve disease. J Cardiovasc Magn Reson 2018;20:28.

26. Hillebrand M, Koschyk D, Ter Hark P, et al. Diagnostic accuracy study of routine echocardiography for bicuspid aortic valve: a retrospective study and meta-analysis. Cardiovasc Diagn Ther 2017;7:367-79.

27. Jilaihawi H, Chen M, Webb J, et al. A Bicuspid Aortic Valve Imaging Classification for the TAVR Era. JACC Cardiovasc Imaging 2016;9:1145-58.

28. Seaman C, Akingba AG, Sucosky P. Steady flow hemodynamic and energy loss measurements in normal and simulated calcified tricuspid and bicuspid aortic valves. J Biomech Eng 2014;136.

29. Baumgartner H, Falk V, Bax JJ, et al. 2017 ESC/EACTS Guidelines for the management of valvular heart disease. Eur Heart J 2017;38:2739-91.

30. Baumgartner H. The 2017 ESC/EACTS guidelines on the management of valvular heart disease : What is new and what has changed compared to the 2012 guidelines? Wien Klin Wochenschr 2018;130:168-71.

31. De Backer J, Renard M, Campens L, et al. Marfan Syndrome and Related Heritable Thoracic Aortic Aneurysms and Dissections. Curr Pharm Des 2015;21:4061-75.

32. Disha K, Espinoza A, Rouman M, et al. Long-Term Recovery of Reduced Left Ventricular Ejection Fraction after Aortic Valve Replacement in Patients with Bicuspid Aortic Valve Disease. Thorac Cardiovasc Surg 2016;64:418-26.

33. Disha K, Rouman M, Secknus MA, et al. Are normal- sized ascending aortas at risk of late aortic events after aortic valve replacement for bicuspid aortic valve disease? Interact Cardiovasc Thorac Surg 2016;22:465-71.

34. Bouhout I, Stevens LM, Mazine A, et al. Long-term outcomes after elective isolated mechanical aortic valve replacement in young adults. J Thorac Cardiovasc Surg 2014;148:1341-6.e1.

35. Puskas J, Gerdisch M, Nichols D, et al. Reduced anticoagulation after mechanical aortic valve replacement: interim results from the prospective randomized on- $\mathrm{X}$ valve anticoagulation clinical trial randomized Food and Drug Administration investigational device exemption trial. J Thorac Cardiovasc Surg 2014;147:1202-10; discussion 1210-1.

36. van Geldorp MW, Eric Jamieson WR, Kappetein AP, et al. Patient outcome after aortic valve replacement with a mechanical or biological prosthesis: weighing lifetime anticoagulant-related event risk against reoperation risk. J Thorac Cardiovasc Surg 2009; 137:881-6, 886e1-5.

37. Rahimtoola SH. Choice of prosthetic heart valve in adults an update. J Am Coll Cardiol 2010;55:2413-26.

38. Glaser N, Jackson V, Holzmann MJ, et al. Aortic valve replacement with mechanical vs. biological prostheses in patients aged 50-69 years. Eur Heart J 2016;37:2658-67.

39. Aicher D, Fries R, Rodionycheva S, et al. Aortic valve repair leads to a low incidence of valve-related complications. Eur J Cardiothorac Surg 2010;37:127-32.

40. Aicher D, Kunihara T, Abou Issa O, et al. Valve configuration determines long-term results after repair of the bicuspid aortic valve. Circulation 2011;123:178-85.

41. Borger MA, Preston M, Ivanov J, et al. Should the ascending aorta be replaced more frequently in patients with bicuspid aortic valve disease? J Thorac Cardiovasc Surg 2004;128:677-83.

42. European Society of Gynecology (ESG); Association for European Paediatric Cardiology (AEPC); German Society for Gender Medicine (DGesGM), et al. ESC Guidelines on the management of cardiovascular diseases during pregnancy: the Task Force on the Management of Cardiovascular Diseases during Pregnancy of the European Society of Cardiology (ESC). Eur Heart J 2011;32:3147-97.

43. Niwa K, Perloff JK, Bhuta SM, et al. Structural abnormalities of great arterial walls in congenital heart disease: light and electron microscopic analyses. Circulation 2001;103:393-400.

44. Koller M, Rothlin M, Senning A. Coarctation of the aorta: review of 362 operated patients. Long-term follow- 
up and assessment of prognostic variables. Eur Heart J 1987;8:670-9.

45. Cohen M, Fuster V, Steele PM, et al. Coarctation of the aorta. Long-term follow-up and prediction of outcome after surgical correction. Circulation 1989;80:840-5.

46. Swan L, Wilson N, Houston AB, et al. The long-term management of the patient with an aortic coarctation repair. Eur Heart J 1998;19:382-6.

47. Haji Zeinali AM, Sadeghian M, Qureshi SA, et al. Midterm to long-term safety and efficacy of self-expandable nitinol stent implantation for coarctation of aorta in adults. Catheter Cardiovasc Interv 2017;90:425-31.

48. Shah L, Hijazi Z, Sandhu S, et al. Use of endovascular stents for the treatment of coarctation of the aorta in children and adults: immediate and midterm results. J Invasive Cardiol 2005;17:614-8.

Cite this article as: Sinning C, Zengin E, Kozlik-Feldmann R, Blankenberg S, Rickers C, von Kodolitsch Y, Girdauskas E. Bicuspid aortic valve and aortic coarctation in congenital heart disease-important aspects for treatment with focus on aortic vasculopathy. Cardiovasc Diagn Ther 2018;8(6):780-788. doi: 10.21037/cdt.2018.09.20
49. Roifman I, Therrien J, Ionescu-Ittu R, et al. Coarctation of the aorta and coronary artery disease: fact or fiction? Circulation 2012;126:16-21.

50. van den Hoven AT, Duijnhouwer AL, Eicken A, et al. Adverse outcome of coarctation stenting in patients with Turner syndrome. Catheter Cardiovasc Interv 2017;89:280-7.

51. Hager A, Kanz S, Kaemmerer H, et al. Coarctation Long-term Assessment (COALA): significance of arterial hypertension in a cohort of 404 patients up to 27 years after surgical repair of isolated coarctation of the aorta, even in the absence of restenosis and prosthetic material. J Thorac Cardiovasc Surg 2007;134:738-45.

52. Beauchesne LM, Connolly HM, Ammash NM, et al. Coarctation of the aorta: outcome of pregnancy. J Am Coll Cardiol 2001;38:1728-33. 